\title{
The production of viscoelastic capillary jets with gaseous flow focusing
}

\author{
A. Ponce-Torres ${ }^{\mathrm{a}, \mathrm{b}}$, J.M. Montanero ${ }^{\mathrm{a}, \mathrm{b}}$, E.J. Vega ${ }^{\mathrm{a}, \mathrm{b}, *}$, A.M. Gañán-Calvo ${ }^{\mathrm{c}}$ \\ a Depto. de Ingeniería Mecánica, Energética y de los Materiales, Universidad de Extremadura, Badajoz E-06006, Spain \\ ${ }^{\mathrm{b}}$ Instituto de Computación Científica Avanzada (ICCAEx), Universidad de Extremadura, Badajoz E-06006, Spain \\ ${ }^{c}$ Depto. de Ingeniería Aeroespacial y Mecánica de Fluidos, Universidad de Sevilla, Sevilla E-41092, Spain
}

\section{A R T I C L E I N F O}

\section{Article history:}

Received 29 April 2015

Revised 28 October 2015

Accepted 12 January 2016

Available online 21 January 2016

\section{Keywords:}

Gaseous flow focusing

Viscoelastic microjet

Jetting regime

Pull-out instability

\begin{abstract}
A B S T R A C T
The production of viscoelastic capillary jets with gaseous flow focusing is studied experimentally. In this technique, the liquid is injected at a constant flow rate through a feeding capillary located in front of the discharge orifice. A gas stream coflows with the jet across the orifice driven by a constant pressure drop. The gas stream sucks and drags the liquid, reducing the jet's diameter well below the orifice diameter. Because of the rheological nature of the liquid, this focusing phenomenon differs from the Newtonian one in several regards. For given values of the polymer concentration, the injected flow rate, and the applied pressure drop, there is an interval of the capillary-to-orifice distance for which the jetting regime is reached. Outside that interval, the jet either suffers from the pull-out instability or breaks up before reaching the discharge orifice. Significant free surface oscillations can be observed in most of the jetting realizations. This oscillatory behavior is caused by a transient die swell effect which continuously appears right at the capillary exit. Ejection interrupts because the jet bulges to such an extent that the free surface pinches. Because of the stabilizing effect of the polymeric contribution to the axial stress, micrometer filaments with lengths up to $1 \mathrm{~cm}$ and Weber numbers on the order of $10^{-4}$ can be produced in front of the discharge orifice. The shear viscous stresses exerted on the emitted jet by the gas stream beyond the discharge orifice prevent the macromolecule recoiling. The resulting extensional viscosity inhibits the break-up process, and thus very long jets are produced.
\end{abstract}

(c) 2016 Elsevier B.V. All rights reserved.

\section{Introduction}

The controlled production of jets on the micrometer scale is of great interest in very diverse fields, such as pharmacy [1], biotechnology [2,3], industrial engineering [4], and the food and agriculture industry [5]. In the Newtonian regime, the produced jets break up into droplets with diameters of the same order of magnitude as that of the jet due to the Rayleigh capillary instability [6]. In this way, relatively monodisperse collections of droplets can be obtained from many jetting realizations to build, for instance, functional materials for health care and pharmacy [1]. On the other side, a variety of physical-chemical processes are used to solidify the produced microjets before their breakup. In particular, viscous liquids are continuously stretched and subsequently solidified in the jetting mode to form sub-millimeter glass or silk fibers [7],

\footnotetext{
* Corresponding author at: Depto. de Ingeniería Mecánica, Energética y de los Materiales, Universidad de Extremadura, E-06006 Badajoz, Spain. Tel.: +34 924 289600; fax: +34924289601.

E-mail address: ejvega@unex.es (E.J. Vega).
}

with obvious applications in the telecommunications and textile industries, respectively.

Many of the above mentioned applications involve the processing of polymeric viscoelastic liquids, where the interactions between the processing conditions and the fluid rheology play a fundamental role. These interactions alter fundamentally the dynamical response of the system, and complicate considerably the analysis of the problem. Nevertheless, rheology becomes more tractable when dealing with Boger fluids [8]. They are dilute polymer solutions in solvents with a sufficiently high viscosity for elastic stresses to be measurable. In addition, Boger fluids exhibit a constant viscosity for a wide range of shear rates (shear thinning can be neglected), so that the elastic effects can be separated from the viscous ones. The Oldroyd-B constitutive equation [9] provides reasonably accurate predictions for these viscoelastic liquids under certain conditions.

Several methods have been proposed to form micro- and nano-meter viscoelastic jets. Among them, electrospinning [10] is very popular because it can be applied to the mass production of one-by-one continuous nanofibers from various polymers. 
However, this method makes use of intense electric fields applied to the polymeric solution, which imposes additional constraints on the liquid electric properties. Micrometer viscoelastic jets have been produced by purely mechanical or hydrodynamic means. In the melt spinning process [11], the liquid is extruded through a small orifice and taken up at a speed higher than the mean extrusion velocity. The resulting filament suffers from self-sustained oscillations when the ratio of the take-up speed to the extrusion one exceeds a critical value close to 20 . This is the so-called draw resonance instability $[11,12]$, which limits considerably both the production rate and the minimum fiber diameter. Selective withdrawal has also been applied to polymer solutions to produce micrometer jets [13]. In this case, an outer liquid stream is forced to cross an orifice located in front of a viscoelastic bath. Both pressure and viscous forces collaborate to form and stretch a liquid meniscus until a very thin jet tapers from its very tip. There are other examples where hydrodynamic forces have also been applied to withdraw fibers with sizes ranging from the millimeter down to the nanometer scale. Viscoelastic filaments have been ejected in a co-axial atomizer to measure their rheological properties [14]. Benavides et al. [15] torn out nanofibers by exposing a polymeric pendant drop to a high-speed gas jet. In the melt blowing (pneumatic melt spinning) method, a molten polymer stream is drawn by two converging air jets directed symmetrically on both sides of a two-slot die [16,17].

Flow focusing $[18,19]$ has become a very popular method to produce Newtonian sub-millimeter jets by strictly hydrodynamic means. In this technique, a meniscus hangs on a feeding capillary through which liquid is injected at a constant flow rate. An outer fluid stream focuses and stretches the meniscus in front of an orifice. The meniscus tapers a thin jet which coflows with the outer stream through the orifice. In the original axisymmetric flow focusing configuration [18], the outer medium was a high-speed gaseous current driven by an applied pressure drop. This configuration was subsequently adapted to the 2D topology [19] to form jets coflowing with an outer liquid stream, which boosted its application in microfluidics [1]. In the two cases, the capillary-to-orifice distance and their diameters are of the same order of magnitude, and the whole emitted jet is influenced by the focusing effect $[19,20]$.

Use has been made of the 2D liquid-liquid flow focusing configuration to produce micrometer jets of Boger fluids [21-26]. For weak elastic effects, the thread pinch-off is initiated by an inertiacapillary mechanism, and followed by an elastocapillary regime [22]. As the polymer relaxation time increases, the filament dynamics becomes controlled primarily by the elastocapillary mechanism, and both the thread length and pinch-off time increase [22]. The flow regimes [25,26], size of the resulting drops [21,23], surfactant effects [24], or appearance of flow asymmetries [27] have been analyzed both numerically and experimentally.

Axisymmetric flow focusing has been applied to form viscoelastic jets focused with an oil phase [28]. A dripping-to-jetting transition was observed for sufficiently high values of the injected flow rate. This transition was explained in terms of the balance between the destabilizing capillary force, and the stabilizing stress associated with the polymer elongation [29]. An effective value of this stress was estimated considering the polymer elongation produced by both the Poiseuille-type flow in the feeding capillary [30], and the subsequent filament stretching in the focusing region. In all the experiments, the tapering meniscus remained anchored to the edge of the feeding capillary end.

To the best of our knowledge, the focusing of viscoelastic jets with a gaseous stream has not as yet been considered. In this paper, we will study experimentally the axisymmetric gaseous flow focusing method to produce micrometer jets of Boger liquids. This technique is not restricted to these liquids, and can also be applied to other non-Newtonian fluids. Fig. 1 sketches the

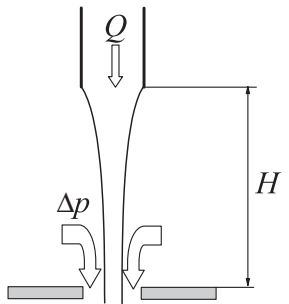

Fig. 1. Sketch of the fluid configuration analyzed in this work.

fluid configuration analyzed in this work. A cylindrical feeding capillary, long as compared to its diameter, is located in front of the discharge orifice, whose diameter is of the same order of magnitude as that of the capillary. The viscoelastic liquid is injected through the feeding capillary at a constant flow rate $Q$, while a gas stream crosses the discharge orifice driven by a constant pressure drop $\Delta p$. If the distance $H$ between the feeding capillary and the discharge orifice lies within a certain range, then a liquid jet forms attached to the edge of the capillary end. The gas stream sucks and drags the liquid, which reduces the jet's diameter down to values much smaller than that of the orifice. Both the liquid jet and the coflowing gas stream cross that orifice. Due to the rheological character of the liquid, this focusing phenomenon differs substantially from that occurring in the Newtonian regime. In particular, the appropriate values for the capillary-to-orifice distance $H$ are much larger than their counterparts in the classical flow focusing configuration. The aerodynamic focusing effect is confined within a region very small as compared with the jet's length, and thus most of the liquid jet flows freely, with no significant interaction with the ambient. The instability mechanisms preventing steady jetting are different from those of the Newtonian mode as well. The polymeric stress stabilizes the liquid thread, which allows one to form jets with virtually zero Weber numbers.

In this paper, attention will be paid mainly to the long (on the order of millimeters) jets formed between the feeding capillary and the discharge orifice. We will determine the conditions for the jetting regime to be reached, and elucidate the instability mechanisms limiting that regime. Here, we review some of the candidates for those mechanisms. The first one is probably the capillary instability [28]. Surface tension makes waves grow over the jet's surface until they eventually pinch the interface. Interestingly, not only the absolute instability [31] but also the convective one might prevent the system from reaching the jetting mode. If perturbations convected downstream pinched the interface in front of the orifice, the suction effect would be interrupted, the filament ejection would cease, and the jetting regime would not be recovered. This phenomenon does not occur in the Newtonian case, where capillary waves grow always beyond the discharge orifice [20].

Capillary instability is a dangerous enemy that can be defeated by the elastic axial stress though. It is well-known that the stress associated with the polymer stretching results in a sharp increase of the extensional viscosity, which partially inhibits the surface tension instability $[32,33]$. The Poiseuille-type flow in the feeding capillary stretches the polymers in the first place. If the jet evolved at a constant speed (diameter), then polymers would relax to their coiling state at distances from the capillary end of the order of its diameter [34-36]. On the contrary, the jet acceleration caused by the co-flowing gas stream next to the orifice keeps the polymer stress unrelaxed or even increases its magnitude.

Lateral (whipping) oscillations may appear in jets coflowing with a much higher speed stream [37,38]. In this case, surface tension has a stabilizing effect, and the destabilizing mechanism is aerodynamic: a perturbation at the interface causes the coflowing fluid to accelerate as it passes a crest, lowering the pressure at that 


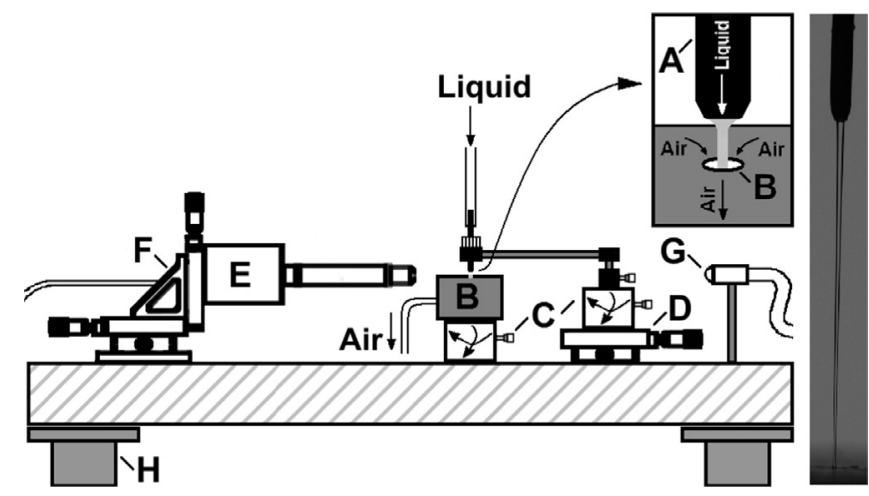

Fig. 2. (Left) Experimental setup: (A) capillary, (B) cell orifice, (C) orientation system, (D) translation stage, (E) camera, (F) triaxial translation stage, (G) optical fiber, and $(\mathrm{H})$ anti-vibration isolation system. (Right) Jet of a PAA solution with $c=1000 \mathrm{ppm}$ produced at a flow rate $Q=4.5 \mathrm{ml} / \mathrm{h}$.

point and encouraging the crest to increase in size. A natural question is whether whipping may play a significant role in our fluid configuration. Experiments have shown that this is the case when focusing Newtonian liquids inside a converging nozzle [39], where the gas stream exhibits an important axial momentum in the focusing region. On the contrary, whipping is confined behind the discharge orifice in the classical flow focusing configuration considered in this work $[20,40]$, because the radial gas flow in front of the orifice constitutes an hydrodynamic barrier for lateral perturbations.

Another possible source of instability is the filament pull-out observed and described in fiber spinning [41-43]. If the filament is stretched by a sufficiently high spinning force, it can be pulled out of the feeding capillary end. The balance between the spinning force and the normal stress in the feeding capillary determines the new position of the meniscus inside the capillary. The meniscus may remain still in the capillary or oscillate around the equilibrium position [43]. Finally, one might also contemplate other instability mechanisms such as the draw resonance mentioned above [11] or the "melt fracture" [44]. It must be noted that draw resonance is linked to the prescription of the jet's velocity somewhere downstream [45]. This condition does not apply to flow focusing, and therefore this phenomenon is not expected in our case.

In this work, we will examine experimentally both the stability and the forces characterizing the jetting regime in gaseous flow focusing of viscoelastic jets. The experimental procedure and rheological properties of the working liquids are described in Section 2. The experimental data are presented and analyzed in Section 3. The paper closes with some conclusions and remarks in Section 4.

\section{Materials and methods}

Fig. 2 shows the experimental setup. The liquid was injected at a constant flow rate $Q$ by a stepper motor through a steel capillary (A), $3.5 \mathrm{~cm}$ in length and $200 \mu \mathrm{m}$ in diameter, with a sharpened end. The capillary end was located in front of an orifice (B) drilled in the upper face of a stainless steel cell. The orifice was $200 \mu \mathrm{m}$ in diameter and $500 \mu \mathrm{m}$ in thickness. A negative gauge pressure $\Delta p$ was applied inside the cell by using a suction pump. We utilized a high-precision orientation system (C) and a translation stage (D) to ensure the correct alignment of the flow focusing elements, and to set the capillary-to-orifice distance $H$. The viscoelastic jet was formed in the open air due to the action of the air stream suctioned through the cell orifice. Digital images were acquired with two cameras (E) with optical axes perpendicular to each other, and equipped with lenses of very different magnifications. The camera with the largest magnification could be displaced both horizontally and vertically using a triaxial translation stage (F) to focus part of the liquid jet, while the other camera allowed us to acquire images of the entire filament. In this way, the jet's radius $R$ (on the order of tens of microns) and the capillary-to-orifice distance $H$ (on the order of millimeters) could be measured simultaneously. The fluid configuration was illuminated from the two back sides (in front of the two cameras) by cool white light provided by two optical fibers $(G)$ connected to light sources. All these elements were mounted on an optical table with a pneumatic anti-vibration isolation system $(\mathrm{H})$ to damp the vibrations coming from the building.

Experiments were conducted at $24 \pm 2{ }^{\circ} \mathrm{C}$. We first located the feeding capillary at a distance from the cell orifice on the order of the orifice diameter. Then, we set the pressure drop $\Delta p$ mbar in the air stream. A liquid flow rate $Q$ was injected through the capillary. After a short transient regime, the capillary ejected a liquid filament which crossed the orifice driven by the air current. We increased progressively the capillary-to-orifice distance $H$ while keeping $\Delta p$ and $Q$ constant. In order to reach the jetting regime, one must follow strictly the above sequence. In fact, if one starts from a $H$ value much larger than the orifice diameter, then the air stream cannot establish the elastic tension in the liquid thread, and the jet does not form. The procedure described above was repeated for different flow rates $Q$ applied pressure drops $\Delta p$, and three polymer solutions. Images of the liquid jet were acquired in the course of the experiments. The free surface position was determined by processing the images with a super-resolution technique at the sub-pixel level [46].

To visualize the outcome of the present technique, some experiments were conducted with a flow focusing nebulizer. In this case, the viscoelastic jet was focused in a glass micronozzle $[2,39]$ by an air stream driven by a positive pressure drop. The nebulizer emitted the jet into the ambient through a discharge orifice $500 \mu \mathrm{m}$ in diameter. This configuration allowed us to acquire sharp images of the jet beyond the discharge orifice.

We examined the behavior of three solutions of poly(acrylic acid) (PAA) $\left(\mathrm{Mw}=18 \times 10^{6} \mathrm{~g} / \mathrm{mol}\right)$ in pure distilled water with concentrations $c=250500$, and $1000 \mathrm{ppm}$. Stock solutions were prepared by dissolving the polymer in a solvent by agitation at very low speeds in order to avoid the breakage of the polymer chains. The dependence of the solution shear viscosity $\mu$ and first viscometric function $\Psi_{1}$ upon the shear rate $\dot{\gamma}$ was measured with a rheometer PHysica MCR 301. The results are displayed in Fig. 3. The polymer relaxation time $\lambda$ was measured with the extensional rheometer HAAKE CaBER 1 by applying the slow retraction method [47]. The values were $\lambda=20,60$ and $140 \mathrm{~ms}$ for 250,500 , and $1000 \mathrm{ppm}$, respectively. The surface tension $\gamma$ was measured with the TIFA method [48]. We verified that $\gamma \simeq 72 \mathrm{mN} / \mathrm{m}$ and $\rho \simeq 997$ in all the cases, i.e., these two properties did not depended significantly on the polymer concentration.

\section{Results}

The gaseous flow focusing of the three viscoelastic liquids described in Section 2 behaves as follows. When the feeding capillary is located next to the discharge orifice, the liquid meniscus detaches from the edge of the capillary end, and climbs over the inner capillary wall. The meniscus either reaches an equilibrium position inside the capillary or oscillates around it. This is the socalled "pull-out" phenomenon already observed in fiber spinning [41-43]. The final position of the triple contact line is essentially determined by the balance between the tensile force originated from the Poiseuille-like flow in the capillary, and that exerted by the emitted jet. A small lateral perturbation makes the jet touch the capillary inner surface. Because of the strong tendency of the liquid to wet steel, the jet remains in contact with the wall 

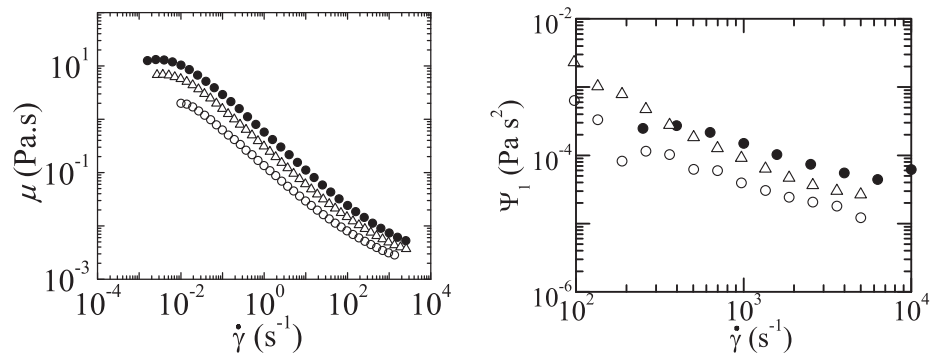

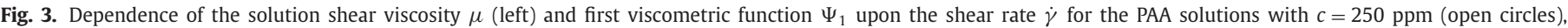
$500 \mathrm{ppm}$ (open triangles) and $1000 \mathrm{ppm}$ (solid circles). The starting points of the curves are determined by the rheometer sensitivity.

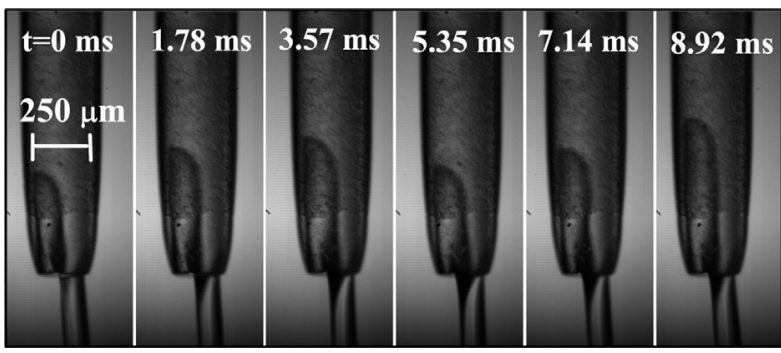

Fig. 4. Sequence of images to illustrate the pull-out phenomenon. The meniscus oscillates around an equilibrium position inside the feeding capillary. The jet was produced with $c=1000 \mathrm{ppm}, Q=40 \mathrm{ml} / \mathrm{h}, \Delta p=250 \mathrm{mbar}$, and $H=0.93 \mathrm{~mm}$ [56].

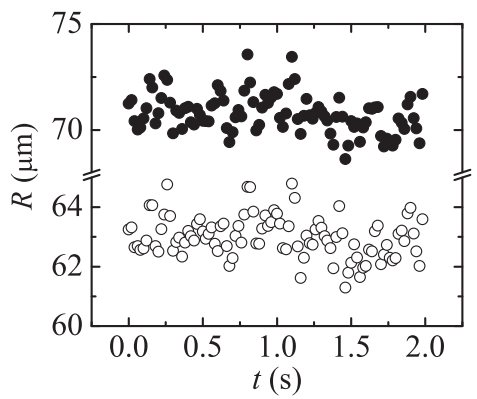

Fig. 5. Time dependence $R(t)$ of the free surface radius at $114 \mu \mathrm{m}$ (solid symbols) and $539 \mu \mathrm{m}$ (open symbols) from the capillary end. The jet was produced with $c=1000 \mathrm{ppm}, Q=5 \mathrm{ml} / \mathrm{h}, \Delta p=250 \mathrm{mbar}$, and $H=6.25 \mathrm{~mm} \mathrm{[56]}$.

while slipping over it. The result is the emission of a steady jet which crosses the discharge orifice driven by the air stream. A sequence of images is displayed in Fig. 4 to illustrate the pull-out phenomenon. In that experiment, the steel capillary was replaced with one made of (transparent) silica.

If one pulls apart gently the feeding capillary and the discharge orifice, the film flowing inside the capillary is unveiled, and becomes part of the jet in the open air. This process continues until the capillary end reaches the meniscus position. Then, the triple contact line anchors to the capillary edge. Contrary to what happens to Newtonian liquids, both the triple contact line and the jet's free surface oscillate. This oscillatory behavior is essentially caused by a transient bulge (die swell effect) which continuously appears right at the capillary exit, probably stimulated by the local relaxation of elastic tension there. The thread bulge is convected downstream, making the entire jet oscillate. For the sake of illustration, Fig. 5 shows the time dependence of the free surface radius for two jet sections.

The oscillation magnitude increases with $H$. There is a maximum capillary-to-orifice distance at which ejection interrupts (Fig. 6). This occurs because the strain rate in that case is not high enough to convect the liquid swelling. Then, the jet

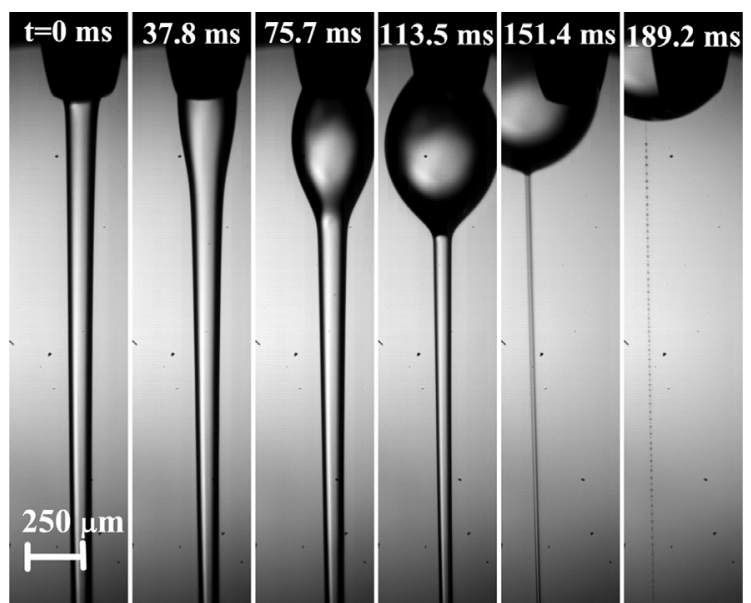

Fig. 6. Sequence of images to illustrate the jet breakage due to the swelling effect. The jet was produced with $c=1000 \mathrm{ppm}, Q=3 \mathrm{ml} / \mathrm{h}, \Delta p=250 \mathrm{mbar}$, and $H=$ $9.1 \mathrm{~mm}[56]$.

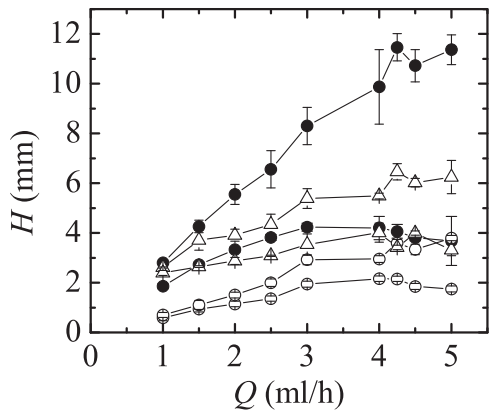

Fig. 7. The minimum and maximum values, $H_{\min }$ and $H_{\max }$, of the capillary-toorifice distance $H$ at which the triple contact line anchors to the capillary edge as a function of the flow rate $O$. The open circles, open triangles and solid circles correspond to the PAA solutions with $c=250,500$ and $1000 \mathrm{ppm}$, respectively [56]. The experiments were conducted with $\Delta p=250$ mbar

continues bulging at the feeding capillary exit, so that a drop forms anchored to the capillary edge. The drop sucks the liquid in the quasi-cylindrical thread hanging on it. The thread radius decreases through an elastocapillary regime similar to that appearing in a capillary breakup rheometer [47,49], which eventually leads to the beads-on-a-string structure and the free surface pinching.

We will call $H_{\min }$ and $H_{\max }$ the capillary-to-orifice distances at which the triple contact line anchors to the capillary edge for the first time, and ejection interrupts, respectively. Steady jetting with pull-out is obtained for $H<H_{\min }$, while oscillating jetting is observed for $H_{\min }<H<H_{\max }$. Fig. 7 shows the values of $H_{\min }$ and $H_{\max }$ corresponding to the PAA solutions with $c=250,500$ and $1000 \mathrm{ppm}$, respectively. The lines delimit the parameter regions 


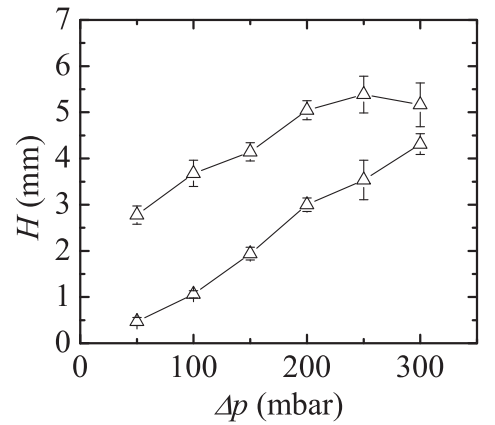

Fig. 8. The minimum and maximum values, $H_{\min }$ and $H_{\max }$, of the capillary-toorifice distance $H$ at which the triple contact line anchors to the capillary edge as a function of the pressure drop $\Delta p$. The experiments were conducted with $c=500 \mathrm{ppm}$ and $Q=3 \mathrm{ml} / \mathrm{h}$.

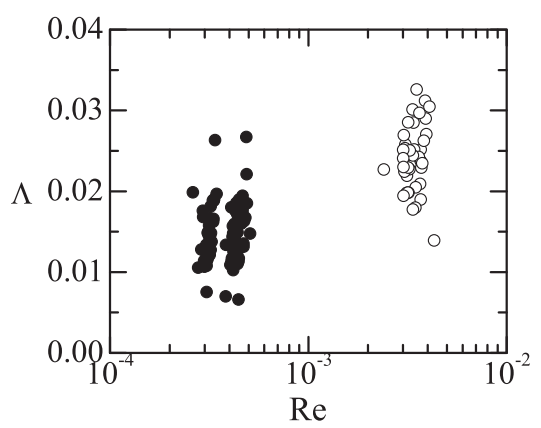

Fig. 9. Reynolds numbers Re and aspect ratios $\Lambda$ in the experiments with $c=$ $250 \mathrm{ppm}$ (open symbols) and $1000 \mathrm{ppm}$ (solid symbols). The experiments were conducted with $\Delta p=250$ mbar.

where the jets anchored to the feeding capillary. Both $H_{\min }$ and $H_{\max }$ increase with $Q$ and so does the difference $H_{\max }-H_{\min }$. The size of the anchorage region increases with $c$ as well. We could not produce jets for $Q<1 \mathrm{ml} / \mathrm{h}$.

Fig. 8 shows the influence of the applied pressure drop $\Delta p$ on both $H_{\min }$ and $H_{\max }$. As $\Delta p$ increases, the stress produced by the gas stream in the discharge orifice increases, and the feeding capillary must be moved further away from the orifice to prevent the filament pull-out. The increase of the strain rate for larger pressure drops favors the convection of the liquid swelling occurring at the capillary end, and therefore the stability limit $H_{\max }$ increases too. The combination of these two effects reduces the range of capillary-to-orifice distances for which the jet remains anchored to the capillary end. The results shown in Figs. 9, 11, and 12 correspond to jetting realizations with anchored contact lines.

Fig. 9 shows the experimental values of the Reynolds number $\operatorname{Re}=\rho v_{0} R_{0} / \mu_{0}$ defined in terms of the jet's radius $R_{0}$ and the mean velocity $v_{0}=Q /\left(\pi R_{0}^{2}\right)$ at the exit of the feeding capillary, and the liquid density $\rho$ and zero-shear viscosity $\mu_{0}$. This latter value was obtained by extrapolating the curves in Fig. 3 to $\dot{\gamma}=0$. As can be observed, Re $\lesssim 10^{-2}$, and thus shear stresses dominate liquid inertia. Therefore, one can assume that viscous radial diffusion of momentum flattens the velocity profile right after the capillary exit, forcing the Poiseuille-like velocity field to evolve into the plug distribution next to the capillary. The figure also shows the values of the aspect ratio $\Lambda=R_{0} / H$. Slender $\left(\Lambda \sim 10^{-2}\right)$ liquid threads form between the feeding capillary and the discharge orifice. Under these conditions, the 1D (slenderness) equation for axial momentum provides an accurate description of the liquid dynamics. Here, we do not integrate the 1D model, but discuss the importance of the different forces acting on the jet in the framework of this approximation.

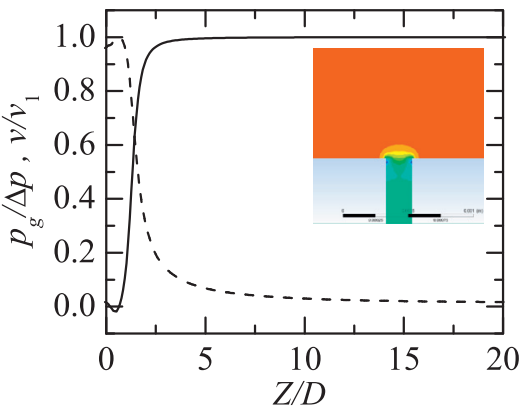

Fig. 10. (Colour online) Velocity $v$ (dashed line) and gauge pressure $p_{g}$ (solid line) distributions along the orifice axis as a function of the distance $Z$ from the center of the orifice. The velocity is divided by its value $v_{1}$ at the orifice inlet section. The simulation was conducted for air driven by a pressure drop $\Delta p=250 \mathrm{mbar}$ and crossing an orifice of diameter $D=200 \mu \mathrm{m}$. The inset shows the pressure field next to the orifice.

In the present flow focusing configuration, the air stream accelerates in a region next to the discharge orifice, with a size much smaller than the liquid thread. In order to illustrate this, we conducted numerical simulations with FLUENT 6.3 of the laminar and incompressible air flow crossing the circular orifice used in our experiments. Fig. 10 shows both the velocity and gauge pressure distributions along the orifice axis as a function of the distance $Z$ from the center of the orifice. As can be observed, the focusing effect is confined within a region of size $Z / D \sim 1$, where $D$ is the orifice diameter. In this region, the force per unit volume exerted by the air stream on the jet scales as $\Delta p / D$ [50]. The size of the focusing region is much smaller than the jet's length, and therefore most of the liquid thread flows freely in front of the discharge orifice, with no significant interaction with the surrounding air.

The 1D momentum equation can be expressed in terms of the following dimensional quantities: the liquid density $\rho$ and surface tension $\gamma$, the solvent (water) viscosity $\mu_{s}\left(\mu_{s}=10^{-3} \mathrm{~kg} / \mathrm{ms}\right)$, the jet's radius $R(z)$ and axial velocity $v(z)$ along the jet axis $z$, as well as the spatial distributions of the axial $\sigma_{z}(z)$ and radial $\sigma_{r}(z)$ polymeric contributions to the total stress. If one neglects the aerodynamic force, then the $1 \mathrm{D}$ equation becomes [37]

$v \frac{d v}{d z}=-\frac{\gamma}{\rho} \frac{d \mathcal{C}}{d z}+\frac{1}{R^{2}} \frac{d}{d z}\left[\left(\sigma_{z}-\sigma_{r}+\frac{3 \mu_{s}}{\rho} \frac{d v}{d z}\right) R^{2}\right]$,

where $\mathcal{C}$ is the free surface local curvature. In order to assess the ranking of the terms appearing in that equation, one scales the radial and axial lengths with $R_{0}$ and $H$, respectively, the jet's velocity with $v_{0}$, and the polymeric stresses with the axial polymeric stress at the feeding capillary exit $\sigma_{z 0}$. This latter quantity can be estimated as $\sigma_{z 0}=\Psi_{1} \dot{\gamma}^{2}$, where $\Psi_{1}(\dot{\gamma})$ is the first viscometric function (Fig. 3), and $\dot{\gamma}$ is the "effective" shear rate characterizing the Poiseuille-like flow in the capillary, i.e., $\dot{\gamma}=4 Q /\left(\pi R_{c}^{3}\right)\left(R_{c}\right.$ is the capillary radius) [28]. The resulting dimensionless equation reads

$$
\underbrace{\hat{R}^{2} \hat{v} \hat{v}^{\prime}}_{\text {I }}=\underbrace{-\mathrm{We}^{-1} \hat{R}^{2} \hat{\mathcal{C}}^{\prime}}_{\text {II }}+\underbrace{\mathrm{We} \mathrm{e}^{-1} \mathrm{Te}\left[\hat{R}^{2}\left(\hat{\sigma}_{z}-\hat{\sigma}_{r}\right)\right]^{\prime}}_{\text {III }}+\underbrace{3 \Lambda \mathrm{Re}_{s}^{-1}\left(\hat{R}^{2} \hat{v}^{\prime}\right)^{\prime}}_{\mathrm{IV}},
$$

where $\hat{R}=R / R_{0}$ and $\hat{v}=v / v_{0}$ are the scaled jet's radius and velocity, respectively, We $=\rho v_{0}^{2} R_{0} / \gamma$ is the Weber number, $\hat{\mathcal{C}}=\mathcal{C} R_{0}$ is the dimensionless free surface local curvature, $\mathrm{Te}=\sigma_{z 0} /\left(\gamma / R_{0}\right)$ is the axial polymeric stress at the feeding capillary exit in terms of the capillary pressure, $\operatorname{Re}_{s}=\rho v_{0} R_{0} / \mu_{S}$ is the Reynolds number based on the solvent viscosity $\mu_{s}$, and $\hat{\sigma}_{r, z}=\sigma_{r, z} / \sigma_{z 0}$ is the scaled polymeric stress field. In addition, the prime denotes the derivative $d / d \hat{z}$ with respect to the scaled axial coordinate $\hat{z}=z / H$. 

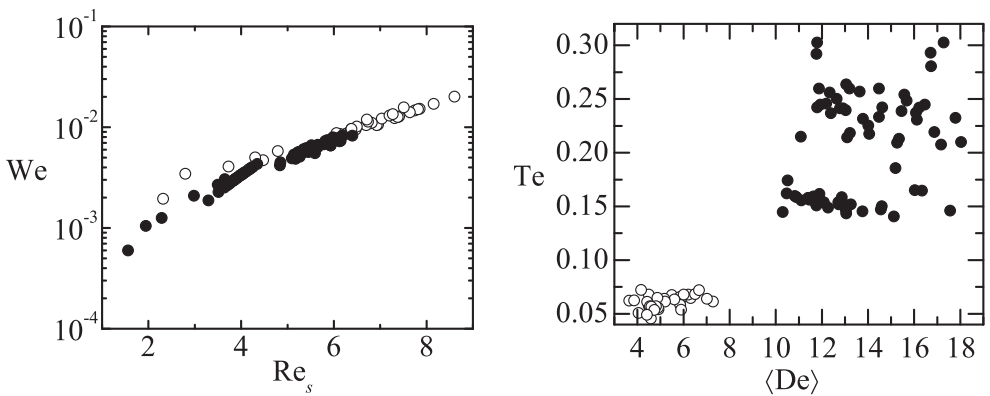

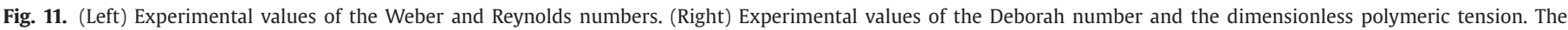
open and solid symbols correspond to the PAA solutions with $c=250$ and $1000 \mathrm{ppm}$, respectively. The experiments were conducted with $\Delta p=250$ mbar.
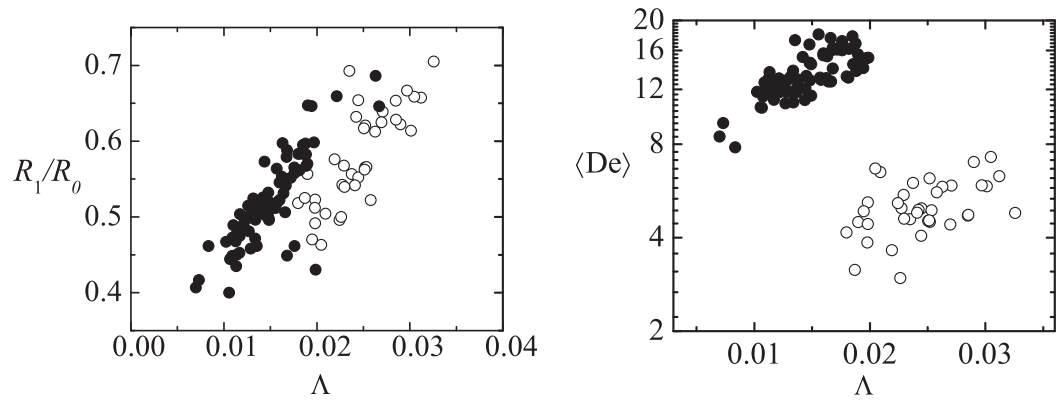

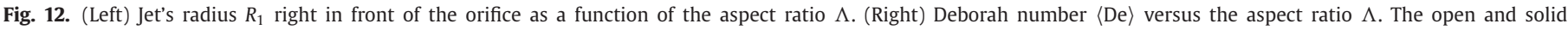
symbols correspond to the PAA solutions with $c=250$ and $1000 \mathrm{ppm}$, respectively. The experiments were conducted with $\Delta p=250$ mbar.

The variations along the jet of its radius and velocity are on the order of $R_{0}$ and $v_{0}$, respectively. Due to the jet slenderness, $\mathcal{C} \simeq$ $1 / R$. Therefore, $\hat{R} \sim \hat{v} \sim \hat{v}^{\prime} \sim \mathcal{C}^{\prime} \sim 1$, and $\left(\hat{R}^{2} \hat{v}^{\prime}\right)^{\prime} \lesssim 1$. Consider now the Deborah number defined as $\mathrm{De}=2 \lambda d v / d z$. According to, for instance, the Oldroyd-B model [9], if De $>1$ the polymeric contribution to the axial stress $\hat{\sigma}_{z}$ grows exponentially, while the radial component $\hat{\sigma}_{r}$ remains inconsequential [37]. We measured in the experiments the average Deborah number $\langle\mathrm{De}\rangle=2 \lambda\left(v_{1}-v_{0}\right) / \mathrm{H}$, where $v_{1}$ is the jet's velocity right in front of the discharge orifice. If $\langle\mathrm{De}\rangle>1$, then one can assume that $\left[\hat{R}^{2}\left(\hat{\sigma}_{z}-\hat{\sigma}_{r}\right)\right]^{\prime}$ grows exponentially throughout the jet.

Fig. 11 shows the experimental values of the Weber, Reynolds, and Deborah numbers, as well as the dimensionless polymeric tension Te. As can be observed, We, $\Lambda \mathrm{Re}_{s}^{-1} \ll 1$ in all the cases, and hence II $\gg I \gg I V$ in Eq. (2). Besides, $\langle\mathrm{De}\rangle>1$ in all the experimental realizations, and thus the term III in Eq. (2) is expected to grow exponentially downstream. One concludes that the jet motion far away from the orifice is driven by the gradient of the polymeric axial stress, while the surface tension may offer a significant resistant force. In other words, the balance of forces reduces approximately to

$\hat{R}^{2} \hat{\mathcal{C}}^{\prime}=\operatorname{Te}\left(\hat{R}^{2} \hat{\sigma}_{z}\right)^{\prime}$.

It must be pointed out the smallness of the Weber number values obtained in our experiments. In fact, capillary jets with We $\simeq 6 \times 10^{-4}$ were produced for the highest polymer concentration and the lowest flow rate. If one compares their kinetic and interfacial energies, one may assert that these jets hang virtually at rest on the feeding capillary. For such small Weber numbers, one must expect the jet to be absolutely unstable [31,51]. This implies that growing waves travel both upstream and downstream over the free surface, preventing perfect steady jetting. As mentioned above, small-magnitude and self-sustained oscillations were observed over the entire liquid domain in the experiments. Absolute instability may partially contribute to the appearance of such oscillations. It must be noted that, contrary to what could happen to Newtonian liquids, those oscillations fail to pinch the free surface due to the stabilizing role played by the polymeric axial stress not only in the linear deformation but also in the nonlinear necking process.

Now, we pay attention to the radius $R_{1}$ of the viscoelastic jet right in front of the orifice. The jet's radius decreases with $\Lambda$ (Fig. 12-left). This result can be interpreted in terms of the effective Deborah number $\langle\mathrm{De}\rangle$ as follows. The Deborah number decreases with $\Lambda$ (Fig. 12-right), which implies that both the average strain rate and the (apparent) extensional viscosity associated with the polymer stretching decrease with $\Lambda$ as well. Therefore, the energy dissipation throughout the liquid thread must decrease with $\Lambda$. Because $\Delta p$ was kept constant in these experiments, the injection of energy into the jet was essentially the same in all the cases. Then, one can conclude that the jet's kinetic energy (radius) in front of the orifice must increase (decrease) as the aspect ratio decreases.

As mentioned in Section 2, use was made of a micronozzle flow focusing nebulizer to acquire sharp images of the ejected viscoelastic filament. Fig. 13 shows snapshots of the jet taken at different distances $Z$ from the discharge orifice. Beyond this orifice, the highspeed air current coflowing with the jet exerts an intense shear viscous stress on the jet free surface, while the pressure force becomes negligible. The shear stress produces strain rates in the liquid which lead to Deborah numbers much greater than unity (De $z 10$ ), and thus the polymer chains remain stretched. As a consequence, the large (apparent) extensional viscosity prevents the jet from breaking up even for distances from the discharge orifice as long as $10^{3}$ times the filament diameter. We have verified that the fluid particle residence time is much shorter than the characteristic time scale for solvent evaporation [52], and therefore the effects of this phenomenon are negligible. In particular, the filament does not solidify. As expected, the air stream triggers whipping instabilities $[37,38]$ in the jet sufficiently far away from the discharge orifice. 


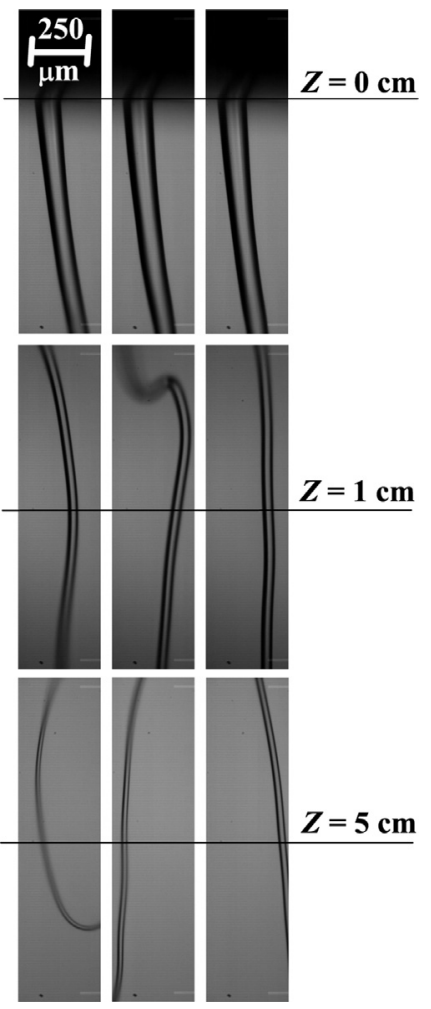

Fig. 13. Images of the viscoelastic jet taken at three distances $Z$ from the discharge orifice. The jet was produced with $c=500 \mathrm{ppm}, \Delta p=250 \mathrm{mbar}, Q=10 \mathrm{ml} / \mathrm{h}$, and $H=0.3 \mathrm{~mm}$.

\section{Conclusions}

We studied experimentally the production of viscoelastic capillary jets with the gaseous flow focusing configuration. The rheological nature of the liquid alters the focusing phenomenon in several important aspects. This technique allows one to form jets in front of the discharge orifice with lengths up to more than one hundred times their radii, and with Weber numbers on the order of $10^{-4}$. Although the focusing region is confined in a small region next to the discharge orifice, the polymeric chains transmit the suction effect upstream throughout the liquid thread. In this way, the resistance offered by surface tension is overcome despite the fact that the kinetic energy is much smaller than the interfacial one. The Weber numbers measured in the experiments are expected to correspond to absolutely unstable jets [51]. In the Newtonian regime, absolute instability always leads to the dripping mode, where the jet cannot even form because the unstable capillary waves travel upstream and pinch the free surface right at the emission point. On the contrary, the rheological character of the liquids inhibits the jetting-to-dripping transition in our experiments, and absolute instability manifests itself as free surface oscillations, at the most.

The jetting regime can be reached within an interval of the capillary-to-orifice distance which strongly depends on the polymer concentration, the flow rate, and the applied pressure drop. Pull-out of the liquid meniscus was found for distances below that interval, while the jet broke up if the distance exceeded the maximum value. Perfect steady jetting was a relatively rare event. In most of the jetting realizations, significant free surface oscillations were observed.

The jet evolution behind the discharge orifice differs substantially from the break-up process extensively analyzed in the literature (see, e.g., $[21,37,49,53,54])$. That process is initially driven by the capillary instability. The subsequent jet deformation stretches the macromolecules in solution, which produces large extensional stresses. These stresses suppress the formation of satellite drops, and lead to the formation of blistering and beads-on-string structures. On the contrary, the shear viscous stresses exerted on the emitted jet by the coflowing gas stream in flow focusing prevent the macromolecules from relaxing. The extensional viscosity associated with the stretched polymers inhibits the break-up process, preserving the liquid filament down to extremely long distances from the discharge orifice.

The competition between surface tension and polymer stresses is the result of a complex interplay between the liquid rheology, the flow rate, and applied pressure drop, as well as the geometrical constraints. The complete characterization of the present technique requires experimentation with a much wider range of experimental conditions, including polymers with varied molecular weights, and different solvents. It must be noted that this technique is not restricted to Boger liquids, and can also be applied to other nonNewtonian fluids. In this case, some of the phenomena described in this paper might significantly change. One might also make use of other liquid feeding means, such as hydrophilic hypodermic needles [55], which may enhance considerably the system stability.

\section{Acknowledgments}

We thank Manuel A. Alves and Patricia C. Sousa for their support in the characterization of the working liquids. Partial support from the Ministry of Science and Education, Junta de Extremadura, and Junta de Andalucía (Spain) through Grants nos. DPI201346485, GR10047, and P08-TEP-04128, respectively, is gratefully acknowledged.

\section{Supplementary material}

Supplementary material associated with this article can be found, in the online version, at 10.1016/j.jnnfm.2016.01.004 .

\section{References}

[1] A.M. Gañán-Calvo, J.M. Montanero, L. Martín-Banderas, M. Flores-Mosquera, Building functional materials for health care and pharmacy from microfluidic principles and flow focusing, Adv. Drug Deliv. Rev. 65 (2013) 1447-1469.

[2] D.P. DePonte, U. Weierstall, K. Schmidt, J. Warner, D. Starodub, J.C.H. Spence, R.B. Doak, Gas dynamic virtual nozzle for generation of microscopic droplet streams, J. Phys. D: Appl. Phys. 41 (2008) 195505

[3] H.N. Chapman, et al., Femtosecond x-ray protein nanocrystallography, Nature 470 (2011) 73-79.

[4] M. Orme, Q. Liu, R. Smith, Molten aluminum micro-droplet formation and deposition for advanced manufacturing applications, Alum. Trans. 3 (2000) 95103.

[5] I. Cohen, H. Li, J.L. Hougland, M. Mrksich, S.R. Nagel, Using selective withdrawal to coat microparticles, Science 292 (2001) 265-267.

[6] L. Rayleigh, On the instability of jets, Proc. Lond. Math. Soc. s1-10 (1878) 4-13.

[7] A. Barrero, I.G. Loscertales, Micro and nanoparticles via capillary flows, Annu. Rev. Fluid Mech. 39 (2007) 89-106.

[8] D.F. James, Boger fluids, Annu. Rev. Fluid Mech. 41 (2009) 129-142.

[9] J.G. Oldroyd, On the formulation of rheological equations of state, Proc. R. Soc Lond. 200 (1950) 523-541.

[10] Z.M. Huanga, Y.Z. Zhang, M. Kotaki, S. Ramakrishna, A review on polymer nanofibers by electrospinning and their applications in nanocomposites, Compos. Sci. Technol. 63 (2003) 2223-2253.

[11] M.M. Denn, Continuous drawing of liquids to form fibers, Annu. Rev. Fluid Mech. 12 (1980) 365-387.

[12] J.R.A. Pearson, M.A. Matovich, Spining a molten threadline. Stability, Ind. Eng. Chem. Fundam. 8 (1969) 605-609.

[13] D. Zhoua, J.J. Feng, Selective withdrawal of polymer solutions: experiments, J. Non-Newton. Fluid Mech. 165 (2010) 829-838.

[14] A. Mansour, N. Chigier, Air-blast atomization of non-newtonian liquids, J. NonNewton. Fluid Mech. 58 (1995) 161-194.

[15] R.E. Benavides, S.C. Jana, D.H. Reneker, Nanofibers from scalable gas jet process, ACS Macro Lett. 1 (2012) 1032-1036.

[16] A. Zachara, Z. Lewandowski, Mathematical modelling of pneumatic melt spinning of isotactic polypropylene. Part I. Modelling of the air jet dynamics, Fibres Text. East. Eur. 16 (2008) 17-23.

[17] D.T. Lohkamp, J.P. Keller, Melt-blowing die using capillary tubes. U.S. Patent US3825379A, 1974 
[18] A.M. Gañán-Calvo, Generation of steady liquid microthreads and micron-sized monodisperse sprays in gas streams, Phys. Rev. Lett. 80 (1998) 285-288.

[19] S.L. Anna, N. Bontoux, H.A. Stone, Formation of dispersions using flow focusing in microchannels, Appl. Phys. Lett. 82 (2003) 364-366.

[20] E.J. Vega, J.M. Montanero, M.A. Herrada, A.M. Gañán-Calvo, Global and local instability of flow focusing: the influence of the geometry, Phys. Fluids 22 (2010) 064105.

[21] C. Zhou, P. Yue, J.J. Feng, Formation of simple and compound drops in microfluidic devices, Phys. Fluids 18 (2006) 092105.

[22] B. Steinhaus, A.Q. Shena, R. Sureshkumar, Dynamics of viscoelastic fluid filaments in microfluidic devices, Phys. Fluids 19 (2007) 073103.

[23] E. Miller, M. Rotea, J.P. Rothstein, Microfluidic device incorporating closed loop feedback control for uniform and tunable production of micro-droplets, Lab Chip 10 (2010) 1293-1301.

[24] W. Lee, L.M. Walker, S.L. Anna, Competition between viscoelasticity and surfactant dynamics in flow focusing microfluidics, Macromol. Mater. Eng. 296 (2011) 203-213.

[25] L. Derzsi, M. Kasprzyk, J.P. Plog, P. Garstecki, Flow focusing with viscoelastic liquids, Phys. Fluids 25 (2013) 092001.

[26] A. Duboin, R. Middleton, F. Malloggi, F. Monti, P. Tabeling, Cusps, spouts and microfiber synthesis with microfluidics, Soft Matter 9 (2013) 3041-3049.

[27] M.S.N. Oliveira, F.T. Pinho, R.J. Poole, P.J. Oliveira, M.A. Alves, Purely elastic flow asymmetries in flow-focusing devices, J. Non-Newton. Fluid Mech. 160 (2009) 31-39.

[28] K.V. Edmond, A.B. Schofield, M. Marquez, J.P. Rothstein, A.D. Dinsmore, Stable jets of viscoelastic fluids and self-assembled cylindrical capsules by hydrodynamic focusing, Langmuir 22 (2006) 9052-9056.

[29] M. Goldin, J. Yerushalmi, R. Pfeffer, R. Shinnar, Breakup of a laminar capillary jet of a viscoelastic fluid, J. Fluid Mech. 38 (1969) 689-711.

[30] R.B. Bird, R.C. Armstrong, O. Hassager, Dynamics of Polymeric Liquids, John Wiley \& Sons, Inc., United States of America, 1987.

[31] P. Huerre, P.A. Monkewitz, Local and global instabilites in spatially developing flows, Annu. Rev. Fluid Mech. 22 (1990) 473-537.

[32] S. Goren, M. Gottlieb, Surface-tension-driven breakup of viscoelastic liquid threads, J . Fluid Mech. 120 (1982) 245-266.

[33] A.C. Ruo, F. Chen, C.A. Chung, M.H. Chang, Three-dimensional response of unrelaxed tension to instability of viscoelastic jets, J . Fluid Mech. 682 (2011) 558-576.

[34] S.J. Gill, J. Gavis, Tensile stress in jets of viscoelastic fluids. i, J. Polym. Sci. 20 (1956) 287-298

[35] A.V. Bazilevskii, V.M. Entov, A.N. Rozhkov, Elastic stresses in capillary jets of dilute polymer solutions, Fluid Dyn. 2 (1985) 3-9.

[36] C. Clasen, J. Bico, V.M. Entov, G.H. McKinley, "Gobbling drops": the jettingdripping transition in flows of polymer solutions, J. Fluid Mech 636 (2009) $5-40$.

[37] J. Eggers, E. Villermaux, Physics of liquid jets, Rep. Prog. Phys. 71 (2008) 036601.

[38] V.M. Entov, A.L. Yarin, The dynamics of thin liquid jets in air, J . Fluid Mech. 140 (1984) 91-111.
[39] A.J. Acero, C. Ferrera, J.M. Montanero, A.M. Gañán-Calvo, Focusing liquid microjets with nozzles, J. Micromech. Microeng. 22 (2012) 065011.

[40] J.M. Montanero, N. Rebollo-Muñoz, M.A. Herrada, A.M. Gañán-Calvo, Global stability of the focusing effect of fluid jet flows, Phys. Rev. E 83 (2011) 036309.

[41] T. Sridhar, R.K. Gupta, Fluid detachment and slip in extensional flows, J. NonNewton. Fluid Mech. 30 (1988) 285-302.

[42] M.J.H. Bulters, H.E.H. Meijer, Analogy between the modelling of pullout in solution spinning and the prediction of the vortex size in contraction flows, J. Non-Newton. Fluid Mech. 38 (1990) 43-80.

[43] C. van der Walt, M.A. Hulsen, A.C.B. Bogaerds, H.E.H. Meijer, M.J.H. Bulters, Stability of fiber spinning under filament pull-out conditions, J. Non-Newton. Fluid Mech. 175-176 (2012) 25-37.

[44] V. Bertola, B. Meulenbroek, C. Wagner, C. Storm, A. Morozov, W. van Saarloos, D. Bonn, Experimental evidence for an intrinsic route to polymer melt fracture phenomena: a nonlinear instability of viscoelastic poiseuille flow, Phys. Rev. Lett. 90 (2003) 114502.

[45] M. Renardy, Draw resonance revisited, J. Phys.: Conf. Ser. 64 (2007) 012016.

[46] E.J. Vega, J.M. Montanero, C. Ferrera, Exploring the precision of backlight optical imaging in microfluidics close to the diffraction limit, Measurement 44 (2011) 1300-1311.

[47] L. Campo-Deaño, C. Clasen, The slow retraction method (SRM) for the determination of ultra-short relaxation times in capillary breakup extensional rheometry experiments, J. Non-Newton. Fluid Mech. 165 (2010) 1688-1699.

[48] M.G. Cabezas, A. Bateni, J.M. Montanero, A.W. Neumann, A new method of image processing in the analysis of axisymmetric drop shapes, Coll. Surf. A 255 (2005) 193-200.

[49] L.E. Rodd, T.P. Scott, J.J. Cooper-White, G.H. Mckinley, Capillary break-up rheometry of low-viscosity elastic fluids, Appl. Rheol. 15 (2005) 12-27.

[50] A.M. Gañán-Calvo, C. Ferrera, J.M. Montanero, Universal size and shape of viscous capillary jets: application to gas-focused microjets, J. Fluid Mech. 670 (2011) 427-438.

[51] A.S. Mohamed, M.A. Herrada, A.M. Gañán-Calvo, J.M. Montanero, Convectiveto-absolute instability transition in a viscoelastic capillary jet subject to unrelaxed axial elastic tension, Phys. Rev. E 92 (2015) 023006.

[52] J. Eggers, Stability of a viscous pinching thread, Phys. Fluids 24 (2012) 072103.

[53] Y. Christanti, L.M. Walker, Surface tension driven jet break up of strainhardening polymer solutions, J. Non-Newton. Fluid Mech. 100 (2001) 9-26.

[54] JJ. Cooper-White, J.E. Fagan, V. Tirtaatmadja, D.R. Lester, D.V. Boger, Drop formation dynamics of constant low-viscosity, elastic fluids, J. Non-Newton. Fluid Mech. 106 (2002) 29-59.

[55] A.J. Acero, N. Rebollo-Muñoz, J.M. Montanero, A.M. Gañán-Calvo, E.J. Vega, A new flow focusing technique to produce very thin jets, J. Micromech. Microeng. 23 (2013) 065009.

[56] See Supplemental Material at [URL will be inserted by publisher] for four videos that show: the pull-out phenomenon, the oscillatory behavior of the jet, the jet breakage due to the swelling effect, and the different stages during the formation and breakup of a viscoelastic jet when one pulls apart gently the feeding capillary and the discharge orifice. 